survival was observed in p-ANCA/MPO patients than c-ANCA-PR3 and ANCA negative patients (Log rank test: $p=0.04$ ).

At univariate analysis of baseline data, deceased patients resulted older at disease onset $(p=0.001)$ with more comorbidities $(p<0.001)$ and presented at diagnosis a higher frequency of respiratory failure ( $p=0.002$, OR 7.1 IC95\% 2.2-22.2) and renal insufficiency ( $p=0.003$, OR $4.795 \% \mathrm{Cl}: 1.6$ to 13.7). No significant differences were noted in term of infections/year, relapses/year and cancer development.

Conclusions: In this large cohort of Italian patients we confirm a higher short and long-term survival in AAV patient than reported in literature. Nevertheless, up to one third of deaths occurred within 6 months after diagnosis and infection diseases resulted the most frequent cause of death. Moreover, our data confirm the prognostic importance of ANCA pattern and the poor outcome of patient with severe lung and renal involvement.

Disclosure of Interest: None declared

DOI: 10.1136/annrheumdis-2018-eular.6974

FRIDAY, 15 JUNE 2018: Tapering and flaring in PsA and SpA

\title{
OP0334 EFFICACY AND SAFETY OF CONTINUING VERSUS WITHDRAWING ADALIMUMAB (ADA) IN MAINTAINING REMISSION IN PATIENTS WITH NON-RADIOGRAPHIC AXIAL SPONDYLOARTHRITIS (NR-AXSPA)
}

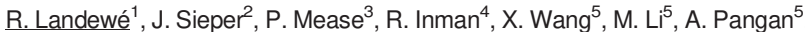
J. Anderson ${ }^{5} .{ }^{1}$ University of Amsterdam, Amsterdam, Netherlands; ${ }^{2}$ Charité Universitätsmedizin Berlin, Berlin, Germany; ${ }^{3}$ Swedish Medical Center and University of Washington, Seattle, USA; ${ }^{4}$ University of Toronto, Toronto, Canada; ${ }^{5}$ AbbVie Inc., North Chicago, USA

Background: It is not known whether TNF blockers can be stopped in nr-axSpA patients (pts) who are in remission.

Objectives: ABILITY-3, reported here, assessed if ADA can be discontinued or should be continued in nr-axSpA pts in sustained remission after a 28-wk openlabel period.

Methods: ABILITY-3 enrolled adult pts diagnosed with nr-axSpA, fulfilling ASAS criteria but NOT modified New York criteria who had objective evidence of active $\mathrm{MRI}$ inflammation in the SI joints or spine or elevated high-sensitivity CRP at screening, active disease at baseline (ASDAS $\geq 2.1$, BASDAI $\geq 4$, total back pain $>4$ ), and inadequate response to $\geq 2$ NSAIDs. Pts who achieved ASDAS inactive disease (ASDAS <1.3) with open-label ADA $40 \mathrm{mg}$ every other wk at wk $16,20,24$, and 28 were randomised to 40 -wk, double-blind PBO (withdrawal) or ADA (continuation) in period 2. Primary efficacy endpoint was proportion of pts who did not experience a flare (ASDAS $\geq 2.1$ at 2 consecutive study visits) during period 2. Secondary endpoints were also assessed up to wk 68 (nonresponder imputation).

Results: Of 673 enrolled pts, 305 (45\%) were randomised to double-blind treatment. A significantly greater proportion of pts treated with ADA vs PBO had no flares $(70 \%$ vs $47 \% ; p<0.001)$ at wk 68 ; relative risk of flare with treatment withdrawal was 1.77. Time to flare analysis showed significantly lower risk of flare for ADA vs PBO (figure 1). At wk 68, significantly greater proportions of ADA vs PBO pts achieved secondary endpoints, except for HAQ-S (table 1). Among pts who received $A D A$ at any time, $77 \%$ reported adverse events (AEs) and $4 \%$ reported a serious $A E$; nasopharyngitis (17\%), upper respiratory tract infection (12\%), worsening of axSpA (9\%), headache (8\%), and diarrhoea (6\%) were the most common. During period 2, incidence of AEs was similar for ADA and PBO (65\% vs $69 \%$ ), incidence of serious AEs was higher for PBO vs ADA (7\% vs $1 \%$ ), and the most common AEs in both the ADA and PBO groups were nasopharyngitis (16\% vs $13 \%$ ), upper respiratory tract infection (13\% vs $8 \%$ ), and worsening of axSpA ( $6 \%$ vs $14 \%$; none serious).

Abstract OP0334 - Table 1 Efficacy outcomes at week 68

\begin{tabular}{lccc} 
Abstract OP0334 - Table 1 Efficacy outcomes at week 68 & & \\
\hline Wk 68, $\mathbf{n}(\%)$ & $\begin{array}{c}\text { ADA } \\
\mathbf{4 0 0} \mathbf{~ m g ~ E O W ) ~} \\
\mathbf{n}=\mathbf{1 5 2}\end{array}$ & $\begin{array}{c}\text { PBO } \\
\mathbf{n}=\mathbf{1 5 3}\end{array}$ & $\begin{array}{c}\boldsymbol{P} \\
\text { Value }\end{array}$ \\
\hline No flare & $106(70)$ & $72(47)$ & $<0.001$ \\
ASDAS ID & $87(57)$ & $51(33)$ & $<0.001$ \\
ASDAS MI & $89(59)$ & $49(32)$ & $<0.001$ \\
ASDAS CII & $102(67)$ & $69(45)$ & $<0.001$ \\
ASAS20 & $107(70)$ & $72(47)$ & $<0.001$ \\
ASAS40 & $100(66)$ & $70(46)$ & $<0.001$ \\
ASAS 5/6 & $87(57)$ & $49(32)$ & $<0.001$ \\
ASAS PR & $64(42)$ & $41(27)$ & 0.005 \\
BASDAI50 & $103(68)$ & $72(47)$ & $<0.001$ \\
Change from baseline in BASFI, LSmean $\pm S E$ & $-3.97 \pm 0.11$ & $-3.51 \pm 0.13$ & 0.007 \\
& $n=111$ & $n=77$ & \\
Change from baseline in HAQ-S, LSmean & $-0.68 \pm 0.04$ & $-0.58 \pm 0.04$ & 0.088 \\
\pm SE & $\mathrm{n}=112$ & $\mathrm{n}=79$ & \\
\hline
\end{tabular}


case of relapse of arthritis, enthesitis or dactylitis under methotrexate monotherapy, golimumab was restarted.

Results: Currently, twenty three of the original $60 \mathrm{pSpA}$ patients included in the CRESPA-trial, completed the 2 year CRESPA-Extension protocol; of these, 21 $(91 \%)$ were in clinical remission at week 104 when methotrexate was added. The mean follow-up period after completion of the extension part, was $80 \pm 28$ w. 5 patients $(24 \%)$ are still in sustained remission $(n=5)$ under methotrexate monotherapy whereas in 16 patients (76\%), golimumab needed to be re-installed because of relapse of disease activity $(n=14)$ or development of adverse events related to methotrexate $(n=2)$. Recurrence of disease was characterised by development of arthritis in all patients with a median of 4 tender and 3 swollen joints. In $50 \%(n=7)$ of the cases, concomitant dactylitis was present. $64 \%(9 / 14)$ were having concomitant psoriasis which was mild since all had a BSA $<5 \%$. The mean time for recurrence was 28,6 weeks. Restarting golimumab treatment promptly restored clinical remission in all patients within 12 weeks.

Conclusions: In patients with pSpA in clinical remission after 2 years of golimumab monotherapy, concomitant administration of methotrexate before discontinuation of the $\mathrm{TNFi}$, did not significantly raise the percentage of patients in biological-free remission. In $76 \%$ of patients, golimumab had to be restarted, underscoring the overall weak efficacy of methotrexate in $\mathrm{pSpA}$.

\section{REFERENCE:}

[1] Carron P, et al. Anti-TNF-induced remission in very early peripheral spondyloarthritis: The CRESPA study. Ann Rheum Dis 2017 Feb 17.

Disclosure of Interest: None declared

DOI: 10.1136/annrheumdis-2018-eular.4287

FRIDAY, 15 JUNE 2018: Prevention of OA: Yes we can!

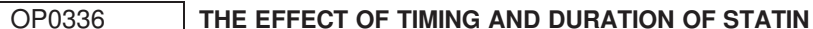 EXPOSURE ON THE RISK OF REVISION FOLLOWING TOTAL HIP OR KNEE ARTHROPLASTY: A POPULATION-BASED COHORT STUDY}

M. Cook ${ }^{1}$, A.K. Sorial ${ }^{2}$, M. Lunt ${ }^{1}$, T. Board ${ }^{3}$, T. O'Neill ${ }^{1} .{ }^{1}$ Arthritis Research UK Centre for Epidemiology, THE UNIVERSITY OF MANCHESTER, Manchester ${ }^{2}$ Institute of Genetic Medicine, Newcastle University, Newcastle; ${ }^{3}$ The Centre for Hip Surgery, Wrightington Wigan and Leigh NHS Foundation Trust, Wigan, UK

Background: Total hip/knee replacement (THR/TKR) are safe and effective interventions for the treatment of osteoarthritis. However, around $2.5 \%$ of patients who undergo a THR/TKR in the UK require revision surgery within 5 years. Experimental studies have suggested that statins may have a beneficial effect on bone by promoting osteoblast formation and reducing osteoclastic bone resorption. Statins have been linked to improved strength of the bone-implant interface and may also attenuate the inflammatory response to particulate wear debris and subsequent periprosthetic osteolysis. Observational data suggest that postoperative exposure to statins may reduce the risk of revision arthroplasty. However, the influence of timing of statin exposure on revision risk has not previously been investigated. This may be significant since statins may affect biological processes occurring at different postoperative periods.

Objectives: To determine whether the timing of statin exposure relative to the primary arthroplasty influences the risk of revision arthroplasty. Also to determine whether the duration of exposure is associated with the risk of revision arthroplasty.

Methods: Subjects from the Clinical Practice Research Datalink, a populationbased clinical database, who had THA/TKA from 1988-2016 were included. Cox regression models were used to determine the association between statin exposure and the risk of revision THA/TKA, i) at any time and ii) if first exposed 0-1, 15 , or $>5$ years following THA/TKA. Cox regression was also used to determine the association between total duration of statin exposure $(<1,1-2,2-3,3-4,4-5,>5$ years) and revision risk. The Cox regression models were adjusted for the propensity score for statin exposure in each period, which was calculated using a logistic regression model including demographic factors, selected comorbidities and selected medication. Missing data for covariates were imputed using multiple imputation by chained equations with 10 iterations.

Results: 151305 participants were included. 57003 (37.7\%) were exposed to statins during follow up and 3500 (2.3\%) had revision arthroplasty. In a propensity score adjusted model, exposure to statins was associated with a reduced risk of revision arthroplasty $(\mathrm{HR}(95 \% \mathrm{Cl}) 0.82(0.75,0.90))$. Participants first exposed within 1 year and between 1 and 5 years following THA/TKA (vs unexposed) had a reduced risk of revision arthroplasty $(\mathrm{HR}(95 \% \mathrm{Cl}) 0.82(0.74,0.91)$ and 0.76 $(0.65,0.90)$, respectively), while first exposure $>5$ years following THA/TKA was not associated with revision risk. In relation to duration of statin therapy, participants exposed for more than 5 years in total ( $v s<1$ year) had a reduced risk of revision $(\mathrm{HR}(95 \% \mathrm{Cl}) 0.74(0.62,0.88))$
Conclusions: Statin therapy initiated up to 5 years following THA/TKA may reduce the risk of revision arthroplasty. The mechanisms by which statin therapy is linked with a reduced risk of revision surgery are not completely understood though does not appear to be related solely to an effect on osseointegration of the primary prosthesis, which occurs primarily in the early $(<1$ year) postoperative period.

Acknowledgements: The authors are grateful to the John Charnley Trust and the Three Wishes Foundation for supporting this research.

Disclosure of Interest: None declared

DOI: 10.1136/annrheumdis-2018-eular.366

\section{OP0337 ASSOCIATION BETWEEN METABOLIC SYNDROME AND TRAJECTORIES OF KNEE PAIN: A 10.7-YEAR FOLLOW- UP STUDY}

F. Pan ${ }^{1}$, J. Tian ${ }^{1}$, F. Cicuttini ${ }^{2}$, C. Ding ${ }^{1}$, G. Jones ${ }^{1} .{ }^{1}$ Menzies Institute for Medical Research, University of Tasmania, Hobart, ${ }^{2}$ Department of Epidemiology and Preventive Medicine, Monash University Medical School, Melbourne, Australia

Background: Metabolic syndrome (MetS) has been suggested as having a role in the pathogenesis of osteoarthritis (OA). However, no study has assessed whether MetS and its components are associated with knee pain and its change over time.

Objectives: To identify distinct trajectories of MSP over 10.7 years in an older population, and to examine risk factors for identified trajectories.

Methods: 1099 participants (mean age 63 years) from the population-based Tasmanian Older Adult Cohort study were recruited at baseline. 875, 768 and 563 participants attended years 2.6, 5.1 and 10.7 follow-up, respectively. Demo graphic, psychological, lifestyle and comorbidities data were obtained at baseline. Knee radiographic OA was assessed by X-ray at baseline. Group-based trajectory modelling was applied to identify distinct trajectories of MSP. Multi-nominal logistic regression was used for the analyses with adjustment for potential confounders.

Results: 985 participants were included for the analyses, three pain trajectories were identified: 'Mild pain' (52\%), 'Moderate pain' (33\%) and 'Severe pain' (15\%) with $32 \%$ of participants having Mets. MetS was significantly associated with increased risk of both 'Moderate pain' (relative risk [RR]: 1.47, 95\% confidence interval [Cl]: 1.10 to 1.96$)$ and 'Severe pain' $(2.22,1.54$ to 3.20$)$ relative to 'Mild pain' in univariate analysis. After adjustment for age, sex, smoking, physical activ ity, emotional problems, comorbidities and radiographic OA, central obesity was associated with increased risk of both 'Moderate pain' (1.70, 1.17 to 2.49 ) and 'Severe pain' $(3.28,2.16$ to 4.98$)$, and MetS and its components (hypertriglyceridemia and low HDL) were only associated with increased risk of 'Severe pain' $(p<0.05)$. However, these associations became weak and non-significant after further adjustment for body mass index (BMI), but hypertension became significantly protective with 'Moderate pain' $(0.70,0.50$ to 0.99$)$. Similar associations were found in those with knee OA (RR: 1.70 to 2.75 , all $p<0.05$ ).

Conclusions: The Mets is predominantly associated with knee pain trajectories through central obesity, and hypertriglyceridemia and low HDL can predict 'Severe pain' trajectory in those with Mets. An unexpected inverse association between hypertension and moderate pain trajectory needs a further investigation which may reflect an interaction between blood pressure and pain sensitivity in 'Moderate pain' trajectory.

Disclosure of Interest: None declared

DOI: 10.1136/annrheumdis-2018-eular.2501

\section{FRIDAY, 15 JUNE 2018}

\section{Big data for musculoskeletal research}

\section{OP0338 RELATIONSHIP OF PROVIDER DENSITY ON TOTAL JOINT REPLACEMENT OUTCOMES}

B. Mehta ${ }^{1,2,3}$, J. Szymonifka ${ }^{4}$, S.A. Dey ${ }^{3}$, I.Y. Navarro-Millan ${ }^{2,3}$, L.A. Mandl ${ }^{2,3}$, A. R. Bass ${ }^{2,3}$, L.A. Russell ${ }^{2,3}$, M.L. Parks ${ }^{5,6}$, M.P. Figgie ${ }^{5,6}$, J.T. Nguyen $^{4}$, S. M. Goodman ${ }^{2,3}$. ${ }^{1}$ Columbia Mailman School of Public Health; ${ }^{2}$ Rheumatology, Weill Cornell Medicine; ${ }^{3}$ Rheumatology; ${ }^{4}$ Biostatistics, HOSPITAL FOR SPECIAL SURGERY; ${ }^{5}$ Orthopaedics, Weill Cornell Medicine; ${ }^{6}$ Orthopaedics, Hospital for Special Surgery, New York, USA

Background: The proportion of providers in a geographical location (provide density) has been associated with improved surgical outcomes in hand and wrist surgery, appendicitis and other high-volume procedures, demonstrating the importance of access to care.

Objectives: The purpose of this study is to assess the association of provide density with Total Knee Replacement (TKR) and Total Hip Replacement (THR) outcomes. 